\title{
Integration of Extended Reality and a High-fidelity Simulator in Team-based Simulations for Arrhythmia Scenarios Among Undergraduate Nursing Students
}

\author{
Youngho Lee \\ Mokpo National University \\ Sun Kyung Kim ( $\nabla$ skkim@mokpo.ac.kr) \\ Mokpo National University \\ Hyoseok Yoon \\ Hanshin University \\ Jongmyung Choi \\ Hanshin University \\ Hyesun Kim
}

Chungbuk National University Hospital

\section{Research Article}

Keywords: arrhythmia, smart glasses, extended reality, team-based simulation, nursing, undergraduate

Posted Date: July 27th, 2021

DOI: https://doi.org/10.21203/rs.3.rs-340621/v2

License: (c) (i) This work is licensed under a Creative Commons Attribution 4.0 International License.

Read Full License 


\section{Abstract}

Objective: The purpose of this study was to evaluate the usability and feasibility of smart glasses for team-based simulations constructed using a high-fidelity simulator.

Methods: Two scenarios of patients with arrhythmia were developed to establish the process for interprofessional interaction via smart-glass in 15 hour simulation training. Three to four participants made a team and took roles of either remote supporter and bad-side trainee with smart-glass. Usability, attitudes towards interprofessional health care team and learning satisfaction were assessed. A scale for software evaluation was used to obtain quantitative data and essay questionnaire were used to assess usability and feasibility of current program. Using SPSS (version 25.0), statistical analysis was conducted.

Results: Data were obtained from 31 participants. The current program was given an easy to use $(3.61 \pm 0.95)$ rating, and the users reported feeling confident during use $(3.90 \pm 0.87)$. Participants responded positively to long-term use (3.26 \pm 0.89$)$ and low levels of physical discomfort $(1.96 \pm 1.06)$. The likability of the smart glasses-based simulation was high, with a high rating for satisfaction (4.65 \pm 0.55$)$. The majority $(84 \%)$ of subjects provided favorable responses to their overall experience with high expectations for improving safety in future clinical practice. Key barriers and challenges of the current program include unstable internet connection, poor resolution and display, and physical discomfort of the smart glasses with accessories.

Conclusion: We determined the feasibility and acceptability of smart glasses for interprofessional interaction within a team-based simulation environment. Participants responded favorably toward a smart glasses-based simulation learning environment that would be applicable in clinical settings.

\section{Introduction}

Patients with arrhythmia are in critical condition, and nurses should be prepared for emergency [1]. In an emergency, health providers must have adequate skills to establish and share common knowledge to make informed decisions. Multiple health professionals would have numerous factors in priority; hence, training for optimal interprofessional interactions would ensure the best patient outcome by providing fast but accurate care in a cooperative manner [2]. Knowing the importance of such issues, organizations such as the American Heart Association, Canadian Cardiovascular Society, and European Society of Cardiology $[3,4]$ have provided guidelines for optimal care. These guidelines are best used when patient data are well collected and analyzed comprehensively, enabling care teams to arrive at an optimal decision [5].

Findings from meta-analyses indicate that simulation-based learning is far more effective than traditional theory-based learning, improving competency and problem-solving 7ability [3,6]. Currently, high-fidelity simulators have been widely used in nursing schools which focuses on reproducing symptoms of illnesses so that students can learn how to care for individual patients[3]. High fidelity alone, however, 
does not sufficiently build a clinical situation where diverse health professionals collaborate in a large range of hospital environments.

'Team-based simulation (TBS)' has been introduced to foster collaborative teamwork and mimic possible challenges that may be encountered while working together with colleagues [7-9]. TBS is an approach aimed at establishing a collaborative learning environment in which students become proficient and actively participate in learning team care for complex health problems [9]. Although the benefits of TBS have been well recognized, current nursing simulation-based education is subject to potential limitations and questions concerning the full engagement of all students involved. Due to a high teacher-student ratio and spatial deficit, 4-5 students comprise a group which is unlikely to go through the decision making with proper interaction between members [10].

With the advent of new technology, methodologies for interprofessional interaction have undergone rapid transformation. At present, with the outbreak of COVID-19, interest in virtual communication has never been higher [11]. Wearable devices, such as smart glasses, have been considered as promising, affordable, and easy-to-setup assistive devices for information exchange in healthcare settings owing to its intuitive design, unobtrusiveness, minimal training requirement, and ability to facilitate telemedical consultation [12] as well as general-purpose in-view recognition [13]. Smart glasses display interactive images on the visual field of users by overlaying visual information without significant hinderance of natural vision $[14,15]$. Thus, it is time to build a learning environment that incorporates cutting edge technology into education to enable students to quickly adapt to a rapidly changing clinical environment.

Given the increased accessibility and improved affordability, there is empirical evidence suggesting the use of smart glasses could result in promising results by enhancing the learning process. The educational application of smart glasses in simulation training has shown strong potential in the acquisition of clinical skills and knowledge [16-18]. Provision of supportive information via smart glasses allows students to confidently make decisions during simulations. Furthermore, the availability for realtime discussion and consultation would increase students' willingness to participate in clinical situations and reduce the burden of uncertainty in their abilities. This study aimed to examine whether adaptation to smart glasses would produce beneficial effects on learning outcomes with TBS by improving team dynamics and learning engagement.

\section{Methods}

\section{Simulation Intervention}

The smart glass based TBS consisted of four parts of 1) lecture, 2) students developing algorithm for patient care, 3) team based training and 4) evaluation. Participants had a lecture for EKG analysis, medication and nursing care for patients with arrhythmia (Table 1). Two scenarios of arrhythmia were introduced, and task was given to build step by step algorithm including decision making process. For each scenario, four students in one group, two students take the role of remote supporters who are in charge of sharing information with bedside workers for optimal decision makings. Students were given 
opportunities to have repetitive training using AR with chances to fix their algorithm. The final test was video recorded for expert evaluation. Since the majority students had only little no previous experience of smart glass, a short instruction of this cooperation system was made prior to simulation education.

Table 1. The simulation frame work for team-based simulation based on scenario of patients with arrhythmia

\begin{tabular}{|ll|}
\hline Simulation framework \\
\hline Teamwork & Bedside Trainee \\
& -Sharing patients' problems and complaints in clear and effective manner \\
& Remote navigator \\
& -Clear allocation of roles and facilitation of cooperation \\
\hline Skill & Nursing intervention \\
performance & - Monitoring, EKG, defibrator, oxygen therapy, intravenous cannula, medication, \\
& patient education \\
& - Checking physician order \\
\hline $\begin{array}{l}\text { Decision } \\
\text { making }\end{array}$ & $\begin{array}{l}\text { 1. Knowledge and recognition of the condition (determining the types of arrhythmia) } \\
\text { (patients who require urgent or emergent care) }\end{array}$ \\
3. Effectiveness of medication \\
4. Patient's condition for additional treatment \\
5. Patient's condition for urgent cardioversion \\
Knowledge of arrhythmia \\
Knowledge
\end{tabular}

The framework of the 15-hour simulation program was developed based on a previous study [19] that included seven nursing skills and 4-5 incidents where students were required to perform nursing practice and make clinical decisions (Appendix 1). The smart glasses-based TBS consisted of three parts; 1)participants attended a lecture on EKG analysis, medication, and nursing care for patients with 
arrhythmia. 2)Two scenarios of arrhythmia (a-fib and PSVT) were introduced, and 3) a task was given to build a step-by-step algorithm. For each scenario, four students formed a group and two students took the role of remote supporters who were in charge of sharing information with bedside workers for optimal decision making. The control group attended traditional TBS, in which all students participated in the simulation as bedside trainees without smart glasses.

\section{Development}

Our system is composed of a trainee-side wearable system, supporter-side desktop system, and network server (Figure 1). The trainee-side wearable system consists of Google Glass (GG), Bluetooth earphones, and a small mirror attached to the GG. GG has a front camera, Bluetooth audio, and a touchpad. The supporter-side desktop system consists of a desktop computer and headset, video conferencing application program to monitor the trainee, and image transmission program for sharing image files.

The trainee-side wearable system transmits real-time video and audio captured from the trainee's field of view through GG and receives audio and image transmitted from the supporter-side system. We used Google Glass Enterprise Edition 2 (Glass EE2; Android Oreo8.1). The supporter-side desktop system receives the trainee's video and enables voice communication. We also developed software that can transmit images and text required for training by accessing the web RTC server using Google Chrome.

Our system was installed in two simulation rooms: the emergency intensive care unit and emergency support unit (Figure 2). In the emergency intensive care unit, there were two trainees wearing a traineeside wearable system and high-fidelity patient simulator. In the emergency support unit, there were two remote supporters sitting the supporter-side desktop system. The supporters can use two desktop application programs: Option1 - Image guide and Option2 - Monitoring system. The image guide delivers selected images to the corresponding trainees and transmits real-time video from the smart glasses to the supporter monitor.

\section{High fidelity simulator}

The METIman is a life-like human simulator that physically represents patients. With clinical features including breathing, pulse, heart and lung sounds, various scenarios of adults with heart disease can be simulated. Not only does the simulator show physical symptoms, but the monitor connected to the simulator displays associated signs (e.g., vital signs).

\section{Sample}

The study population included nursing students who attended a baccalaureate nursing program at a university located in $\mathrm{J}$ district, Korea. A total of 32 participants were recruited and data from 31 participants. The purpose of this study was explained to all study participants and written informed consent was obtained.

\section{Instrument}




\section{Usability}

Developed by Ingrassia et al. [20], we revised the questionnaire according to the design and purpose of this study. The survey consisted of 54 questions spanning six categories: user input (12 items), system output (6 items), system usability (17 items), fidelity (8 items), immersivity (4 items), and likability (7 items). Using a 5-point Likert scale, the study participants provided ratings from 1 (strongly disagree) to 5 (strongly agree). There were four additional questions in which study participants rated their overall level of satisfaction from 1 (very dissatisfied) to 4 (very satisfied) regarding the smart glasses device, ease of use, system output, and smart glasses-based simulation.

\section{Attitudes towards the interprofessional health care team}

The Attitudes towards Interprofessional Health Care Teams scale was developed by Heinemann et al. [21] and modified by Curran et al. [22]. This scale consists of 14 items that include the quality of care, time constraints, and teamwork of health professionals. This scale uses a 5-point Likert scale with values ranging from 'strongly disagree' (1) to 'strongly agree' ( 5).

\section{Learning satisfaction}

Satisfaction with the education program was measured using the questionnaire used by $\mathrm{Ji}$ and Chung [23]. The 5-point Likert scores were used, randing from "not at all satisfied" (1) to "very satisfied" (5). The higher the score, the higher the satisfaction with the education program.

\section{Essay questionnaire}

An essay questionnaire was used that asked seven qualitative questions: 1) "How did you find the smart glasses-based emergency simulation in general?"; 2) "Was this program easy to use? What are the points that you thought needed further improvement?"; 3) "Were there any difficulties or constraints when operating the system?"; 4) "Do you think this smart glass-based simulation education would be useful for your future practice?"; 5) "Do you think the smart glass would be useful in the clinical environment?"; 6) "What are the components that require additional technical efforts for active application and continuous use of these smart glasses?"; and 7) "Please add any other comment on this program."

\section{Statistical analysis}

Statistical analyses were performed using SPSS (version 25.0). For demographic data, the frequency, percentage, mean, and standard deviation were calculated. Independent t-tests and chi-squared tests were used to examine the homogeneity between the intervention and control groups. Independent t-tests were used to compared group differences, and statistical significance was set at $p<.05$.

\section{Results}

Table 2. General characteristic of study participants and homogeneity test between groups $(n=61)$ 


\begin{tabular}{|c|c|c|c|c|}
\hline & & $\begin{array}{l}\text { Intervention } \\
(\mathrm{n}=31)\end{array}$ & $\begin{array}{l}\text { Control } \\
(n=30)\end{array}$ & $\begin{array}{l}t / X^{2} \\
\text { (p) }\end{array}$ \\
\hline Age (years) & & $23.9 \pm 1.24$ & $23.7 \pm 2.07$ & $.617(.539)$ \\
\hline \multirow[t]{2}{*}{ Sex } & Female & $23(74.2)$ & $28(93.3)$ & \multirow{2}{*}{$\begin{array}{l}4.075 \\
(.044)\end{array}$} \\
\hline & Male & $8(25.8)$ & $2(6.7)$ & \\
\hline \multirow[t]{3}{*}{ Grade } & Poor & $5(16.1)$ & $3(10)$ & \multirow{3}{*}{$\begin{array}{l}.561 \\
(.756)\end{array}$} \\
\hline & Fair & $20(64.5)$ & $20(66.7)$ & \\
\hline & Good & $6(19.4)$ & $7(23.3)$ & \\
\hline \multirow[t]{3}{*}{ Major satisfaction } & Poor & $0(0)$ & $0(0)$ & \multirow{3}{*}{$\begin{array}{l}.415 \\
(.519)\end{array}$} \\
\hline & Fair & $8(25.8)$ & $10(33.3)$ & \\
\hline & Good & $23(74.2)$ & $20(66.7)$ & \\
\hline \multirow[t]{5}{*}{ Previous use of Augmented reality } & Never used & $14(45.1)$ & $16(53.4)$ & \multirow{5}{*}{$\begin{array}{l}4.085 \\
(.252)\end{array}$} \\
\hline & A few times & $11(35.5)$ & $13(43.3)$ & \\
\hline & Once a month & $4(12.9)$ & $1(3.3)$ & \\
\hline & Once a week & $2(6.5)$ & $0(0)$ & \\
\hline & Every day & $0(0)$ & $0(0)$ & \\
\hline \multirow[t]{5}{*}{ Previous use of smart glasses } & Never used & $20(64.5)$ & $20(66.7)$ & \multirow{5}{*}{$\begin{array}{l}.031 \\
(.860)\end{array}$} \\
\hline & A few times & $11(35.5)$ & $10(33.3)$ & \\
\hline & Once a month & $0(0)$ & $0(0)$ & \\
\hline & Once a week & $0(0)$ & $0(0)$ & \\
\hline & Every day & $0(0)$ & $0(0)$ & \\
\hline
\end{tabular}

Data are presented as the mean \pm standard deviation or number $(\%)$.

The mean age of study participants were 23.9 and 23.7 respectively and the intervention group had higher proportion of male participants $(p=.044)$ (Table 2$)$

Table 3. User input and system output 


\begin{tabular}{|c|c|c|c|c|c|c|}
\hline User input & $\begin{array}{l}\text { Mean } \pm S D \\
(1-5)\end{array}$ & $\begin{array}{l}\text { Strongly } \\
\text { Disagree } \\
n(\%)\end{array}$ & $\begin{array}{l}\text { Disagree } \\
n(\%)\end{array}$ & $\begin{array}{l}\text { Neither } \\
\mathrm{n}(\%)\end{array}$ & $\begin{array}{l}\text { Agree } \\
\mathrm{n}(\%)\end{array}$ & $\begin{array}{l}\text { Strongly } \\
\text { agree } \\
\mathrm{n}(\%)\end{array}$ \\
\hline $\begin{array}{l}\text { I had the right level of } \\
\text { control over what I wanted } \\
\text { to do }\end{array}$ & $3.77 \pm 0.67$ & & $\begin{array}{l}2 \\
(6.5)\end{array}$ & $\begin{array}{l}5 \\
(16.1)\end{array}$ & $\begin{array}{l}22 \\
(71.0)\end{array}$ & $\begin{array}{l}2 \\
(6.5)\end{array}$ \\
\hline $\begin{array}{l}\text { The effect of my } \\
\text { interaction was easy to } \\
\text { predict }\end{array}$ & $3.71 \pm 0.74$ & & $\begin{array}{l}2 \\
(6.5)\end{array}$ & $\begin{array}{l}8 \\
(25.8)\end{array}$ & $\begin{array}{l}18 \\
(58.1)\end{array}$ & $\begin{array}{l}3 \\
(9.7)\end{array}$ \\
\hline $\begin{array}{l}\text { The system did not } \\
\text { behave as I expected }\end{array}$ & $2.45 \pm 1.12$ & $\begin{array}{l}6 \\
(19.4)\end{array}$ & $\begin{array}{l}13 \\
(41.9)\end{array}$ & $\begin{array}{l}5 \\
(16.1)\end{array}$ & $\begin{array}{l}6 \\
(19.4)\end{array}$ & $\begin{array}{l}1 \\
(3.2)\end{array}$ \\
\hline $\begin{array}{l}\text { I could not always achieve } \\
\text { what I wanted the system } \\
\text { to do }\end{array}$ & $2.00 \pm 0.86$ & $\begin{array}{l}9 \\
(29.0)\end{array}$ & $\begin{array}{l}15 \\
(48.4)\end{array}$ & $\begin{array}{l}5 \\
(16.1)\end{array}$ & $\begin{array}{l}2 \\
(6.5)\end{array}$ & \\
\hline $\begin{array}{l}\text { I kept making mistakes } \\
\text { interacting with the } \\
\text { system }\end{array}$ & $2.42 \pm 1.18$ & $\begin{array}{l}9 \\
(29.0)\end{array}$ & $\begin{array}{l}7 \\
(22.6)\end{array}$ & $\begin{array}{l}9 \\
(29.0)\end{array}$ & $\begin{array}{l}5 \\
(16.1)\end{array}$ & $\begin{array}{l}1 \\
(3.2)\end{array}$ \\
\hline $\begin{array}{l}\text { Accurate pointing with } \\
\text { gaze was easy to achieve }\end{array}$ & $2.65 \pm 1.23$ & $\begin{array}{l}5 \\
(16.1)\end{array}$ & $\begin{array}{l}12 \\
(38.7)\end{array}$ & $\begin{array}{l}6 \\
(19.4)\end{array}$ & $\begin{array}{l}5 \\
(16.1)\end{array}$ & $\begin{array}{l}3 \\
(9.7)\end{array}$ \\
\hline $\begin{array}{l}\text { Hand interactions were } \\
\text { difficult to perform }\end{array}$ & $1.81 \pm 1.05$ & $\begin{array}{l}15 \\
(48.4)\end{array}$ & $\begin{array}{l}11 \\
(35.5)\end{array}$ & $\begin{array}{l}2 \\
(6.5)\end{array}$ & $\begin{array}{l}2 \\
(6.5)\end{array}$ & $\begin{array}{l}1 \\
(3.2)\end{array}$ \\
\hline $\begin{array}{l}\text { Interaction with the } \\
\text { system was fast enough }\end{array}$ & $3.29 \pm 1.22$ & $\begin{array}{l}2 \\
(6.5)\end{array}$ & $\begin{array}{l}7 \\
(22.6)\end{array}$ & $\begin{array}{l}8 \\
(25.8)\end{array}$ & $\begin{array}{l}8 \\
(25.8)\end{array}$ & $\begin{array}{l}6 \\
(19.4)\end{array}$ \\
\hline $\begin{array}{l}\text { The system responded too } \\
\text { slowly during interaction }\end{array}$ & $2.03 \pm 0.87$ & $\begin{array}{l}9 \\
(29.0)\end{array}$ & $\begin{array}{l}14 \\
(45.2)\end{array}$ & $\begin{array}{l}6 \\
(19.4)\end{array}$ & $\begin{array}{l}2 \\
(6.5)\end{array}$ & \\
\hline $\begin{array}{l}\text { I would have preferred } \\
\text { alternative interaction } \\
\text { methods }\end{array}$ & $3.00 \pm 1.10$ & $\begin{array}{l}4 \\
(12.9)\end{array}$ & $\begin{array}{l}5 \\
(16.1)\end{array}$ & $\begin{array}{l}10 \\
(32.3)\end{array}$ & $\begin{array}{l}11 \\
(35.5)\end{array}$ & $\begin{array}{l}1 \\
(3.2)\end{array}$ \\
\hline $\begin{array}{l}\text { I found the touch pad was } \\
\text { too sensitive }\end{array}$ & $2.48 \pm 1.09$ & $\begin{array}{l}7 \\
(22.6)\end{array}$ & $\begin{array}{l}9 \\
(29.0)\end{array}$ & $\begin{array}{l}8 \\
(25.8)\end{array}$ & $\begin{array}{l}7 \\
(22.6)\end{array}$ & \\
\hline $\begin{array}{l}\text { I found the input } \\
\text { modalities ideal for the } \\
\text { task to be performed }\end{array}$ & $3.81 \pm 0.70$ & $\begin{array}{l}1 \\
(3.2)\end{array}$ & $\begin{array}{l}8 \\
(25.8)\end{array}$ & $\begin{array}{l}18 \\
(58.1)\end{array}$ & $\begin{array}{l}4 \\
(12.9)\end{array}$ & \\
\hline \multicolumn{7}{|l|}{ System output } \\
\hline $\begin{array}{l}\text { I found the display appropria } \\
\text { for the tasks }\end{array}$ & $3.87 \pm$ & $\begin{array}{l}1 \\
(3.2)\end{array}$ & $\begin{array}{l}3 \\
(9.7)\end{array}$ & $\begin{array}{l}4 \\
(12.9)\end{array}$ & $\begin{array}{l}14 \\
(45.2)\end{array}$ & $\begin{array}{l}9 \\
(29.0)\end{array}$ \\
\hline
\end{tabular}




\begin{tabular}{|c|c|c|c|c|c|c|}
\hline $\begin{array}{l}\text { I felt the display was too limited } \\
\text { to see the contents and carry } \\
\text { out the task }\end{array}$ & $3.39 \pm 1.17$ & $\begin{array}{l}3 \\
(9.7)\end{array}$ & $\begin{array}{l}4 \\
(12.9)\end{array}$ & $\begin{array}{l}6 \\
(19.4)\end{array}$ & $\begin{array}{l}14 \\
(45.2)\end{array}$ & $\begin{array}{l}4 \\
(12.9)\end{array}$ \\
\hline $\begin{array}{l}\text { Screen was not legible because } \\
\text { of light, reflection or glare }\end{array}$ & $2.48 \pm 1.20$ & $\begin{array}{l}8 \\
(25.8)\end{array}$ & $\begin{array}{l}9 \\
(29.0)\end{array}$ & $\begin{array}{l}6 \\
(19.4)\end{array}$ & $\begin{array}{l}7 \\
(22.6)\end{array}$ & $\begin{array}{l}1 \\
(3.2)\end{array}$ \\
\hline $\begin{array}{l}\text { I felt that the display was } \\
\text { flickering too much and } \\
\text { graphics were too unstable }\end{array}$ & $1.94 \pm 0.89$ & $\begin{array}{l}11 \\
(35.5)\end{array}$ & $\begin{array}{l}13 \\
(41.9)\end{array}$ & $\begin{array}{l}5 \\
(16.1)\end{array}$ & $\begin{array}{l}2 \\
(6.5)\end{array}$ & \\
\hline $\begin{array}{l}\text { I thought the words and } \\
\text { symbols on screen were easy to } \\
\text { read }\end{array}$ & $3.68 \pm 1.24$ & $\begin{array}{l}3 \\
(9.7)\end{array}$ & $\begin{array}{l}3 \\
(9.7)\end{array}$ & $\begin{array}{l}3 \\
(9.7)\end{array}$ & $\begin{array}{l}14 \\
(45.2)\end{array}$ & $\begin{array}{l}8 \\
(25.8)\end{array}$ \\
\hline $\begin{array}{l}\text { The quality of the display } \\
\text { affected my performance }\end{array}$ & $3.00 \pm 1.18$ & $\begin{array}{l}4 \\
(12.9)\end{array}$ & $\begin{array}{l}7 \\
(22.6)\end{array}$ & $\begin{array}{l}7 \\
(22.6)\end{array}$ & $\begin{array}{l}11 \\
(35.5)\end{array}$ & $\begin{array}{l}2 \\
(6.5)\end{array}$ \\
\hline
\end{tabular}

The participants reported good level of control over the system overall (mean 3.77, SD 0.67). Some expressed lack of controllability (22.6\%) and preference to alternative interaction methods (38.7\%). Majority of participants (87.1\%) reported appropriateness of display of system. In terms of contents provided via screen of smart-glass, $58.1 \%$ of participants experienced some degree of limitations for seamless performance of tasks.

Table 4. System usability of smart glass based TBS program $(n=31)$ 


\begin{tabular}{|c|c|c|c|c|c|c|}
\hline System Usability Scale (SUS) & $\begin{array}{l}\text { Mean } \pm S D \\
(1-5)\end{array}$ & $\begin{array}{l}\text { Strongly } \\
\text { Disagree } \\
\mathrm{n}(\%)\end{array}$ & $\begin{array}{l}\text { Disagree } \\
n(\%)\end{array}$ & $\begin{array}{l}\text { Neither } \\
\mathrm{n}(\%)\end{array}$ & $\begin{array}{l}\text { Agree } \\
\mathrm{n}(\%)\end{array}$ & $\begin{array}{l}\text { Strongly } \\
\text { agree } \\
\mathrm{n}(\%)\end{array}$ \\
\hline $\begin{array}{l}\text { I would use this program } \\
\text { frequently }\end{array}$ & $3.87 \pm 0.76$ & & $\begin{array}{l}2 \\
(6.5)\end{array}$ & $\begin{array}{l}5 \\
(16.1)\end{array}$ & $\begin{array}{l}19 \\
(61.3)\end{array}$ & $\begin{array}{l}5 \\
(16.1)\end{array}$ \\
\hline $\begin{array}{l}\text { The program was } \\
\text { unnecessarily complex }\end{array}$ & $2.19 \pm 1.14$ & $\begin{array}{l}10 \\
(32.3)\end{array}$ & $\begin{array}{l}11 \\
(35.5)\end{array}$ & $\begin{array}{l}5 \\
(16.1)\end{array}$ & $\begin{array}{l}4 \\
(12.9)\end{array}$ & $\begin{array}{l}1 \\
(3.2)\end{array}$ \\
\hline The program was easy to use & $3.61 \pm 0.95$ & $\begin{array}{l}1 \\
(3.2)\end{array}$ & $\begin{array}{l}3 \\
(9.7)\end{array}$ & $\begin{array}{l}7 \\
(22.6)\end{array}$ & $\begin{array}{l}16 \\
(51.6)\end{array}$ & $\begin{array}{l}4 \\
(12.9)\end{array}$ \\
\hline I needed technical support & $3.90 \pm 0.79$ & & $\begin{array}{l}1 \\
(3.2)\end{array}$ & $\begin{array}{l}8 \\
(25.8)\end{array}$ & $\begin{array}{l}15 \\
(48.4)\end{array}$ & $\begin{array}{l}7 \\
(22.6)\end{array}$ \\
\hline $\begin{array}{l}\text { Various functions were well } \\
\text { integrated }\end{array}$ & $3.77 \pm 0.84$ & & $\begin{array}{l}3 \\
(9.7)\end{array}$ & $\begin{array}{l}6 \\
(19.4)\end{array}$ & $\begin{array}{l}17 \\
(54.8)\end{array}$ & $\begin{array}{l}5 \\
(16.1)\end{array}$ \\
\hline $\begin{array}{l}\text { There were too much } \\
\text { inconsistencies }\end{array}$ & $3.32 \pm 1.25$ & $\begin{array}{l}4 \\
(12.9)\end{array}$ & $\begin{array}{l}3 \\
(9.7)\end{array}$ & $\begin{array}{l}8 \\
(25.8)\end{array}$ & $\begin{array}{l}11 \\
(35.5)\end{array}$ & $\begin{array}{l}5 \\
(16.1)\end{array}$ \\
\hline $\begin{array}{l}\text { The most people would find } \\
\text { this program easy to use }\end{array}$ & $4.06 \pm 0.92$ & $\begin{array}{l}1 \\
(3.2)\end{array}$ & $\begin{array}{l}1 \\
(3.2)\end{array}$ & $\begin{array}{l}3 \\
(9.7)\end{array}$ & $\begin{array}{l}16 \\
(51.6)\end{array}$ & $\begin{array}{l}10 \\
(32.3)\end{array}$ \\
\hline $\begin{array}{l}\text { The program was very } \\
\text { cumbersome to use }\end{array}$ & $2.06 \pm 1.06$ & $\begin{array}{l}11 \\
(35.5)\end{array}$ & $\begin{array}{l}12 \\
(38.7)\end{array}$ & $\begin{array}{l}3 \\
(9.7)\end{array}$ & $\begin{array}{l}5 \\
(16.1)\end{array}$ & \\
\hline $\begin{array}{l}\text { I felt confident using this } \\
\text { program }\end{array}$ & $3.90 \pm 0.87$ & & $\begin{array}{l}2 \\
(6.5)\end{array}$ & $\begin{array}{l}7 \\
(22.6)\end{array}$ & $\begin{array}{l}14 \\
(45.2)\end{array}$ & $\begin{array}{l}8 \\
(25.8)\end{array}$ \\
\hline $\begin{array}{l}\text { I think I need to learn } \\
\text { numerous things before using } \\
\text { this program }\end{array}$ & $3.74 \pm 1.21$ & $\begin{array}{l}2 \\
(6.5)\end{array}$ & $\begin{array}{l}3 \\
(9.7)\end{array}$ & $\begin{array}{l}6 \\
(19.4)\end{array}$ & $\begin{array}{l}10 \\
(32.3)\end{array}$ & $\begin{array}{l}10 \\
(32.3)\end{array}$ \\
\hline Google glass $2^{\text {nd }}$ Edition & & & & & & \\
\hline $\begin{array}{l}\text { The Google Glass is too big } \\
\text { and heavy }\end{array}$ & $1.77 \pm 0.88$ & $\begin{array}{l}15 \\
(48.4)\end{array}$ & $\begin{array}{l}9 \\
(29.0)\end{array}$ & $\begin{array}{l}6 \\
(19.4)\end{array}$ & $\begin{array}{l}1 \\
(3.2)\end{array}$ & \\
\hline $\begin{array}{l}\text { The device needs substantial } \\
\text { mental efforts (concentration) } \\
\text { to operate }\end{array}$ & $2.65 \pm 1.43$ & $\begin{array}{l}10 \\
(32.3)\end{array}$ & $\begin{array}{l}5 \\
(16.1)\end{array}$ & $\begin{array}{l}5 \\
(16.1)\end{array}$ & $\begin{array}{l}8 \\
(25.8)\end{array}$ & $\begin{array}{l}3 \\
(9.7)\end{array}$ \\
\hline $\begin{array}{l}\text { The device needs great } \\
\text { physical efforts to operate }\end{array}$ & $1.94 \pm 1.06$ & $\begin{array}{l}14 \\
(45.2)\end{array}$ & $\begin{array}{l}9 \\
(29.0)\end{array}$ & $\begin{array}{l}4 \\
(12.9)\end{array}$ & $\begin{array}{l}4 \\
(12.9)\end{array}$ & \\
\hline
\end{tabular}




\begin{tabular}{|c|c|c|c|c|c|c|}
\hline $\begin{array}{l}\text { There was high fatigue in arm, } \\
\text { hands and fingers }\end{array}$ & $1.42 \pm 0.72$ & $\begin{array}{l}21 \\
(67.7)\end{array}$ & $\begin{array}{l}8 \\
(25.8)\end{array}$ & $\begin{array}{l}1 \\
(3.2)\end{array}$ & $\begin{array}{l}1 \\
(3.2)\end{array}$ & \\
\hline $\begin{array}{l}\text { There was very high fatigue in } \\
\text { eyes }\end{array}$ & $2.19 \pm 1.17$ & $\begin{array}{l}12 \\
(38.7)\end{array}$ & $\begin{array}{l}7 \\
(22.6)\end{array}$ & $\begin{array}{l}6 \\
(19.4)\end{array}$ & $\begin{array}{l}6 \\
(19.4)\end{array}$ & \\
\hline $\begin{array}{l}\text { There was very high fatigue in } \\
\text { head }\end{array}$ & $1.55 \pm 0.72$ & $\begin{array}{l}18 \\
(58.1)\end{array}$ & $\begin{array}{l}9 \\
(29.0)\end{array}$ & $\begin{array}{l}4 \\
(12.9)\end{array}$ & & \\
\hline $\begin{array}{l}\text { I think I can use the device for } \\
\text { long time }\end{array}$ & $3.26 \pm 0.89$ & & $\begin{array}{l}7 \\
(22.6)\end{array}$ & $\begin{array}{l}11 \\
(35.5)\end{array}$ & $\begin{array}{l}11 \\
(35.5)\end{array}$ & $\begin{array}{l}2 \\
(6.5)\end{array}$ \\
\hline
\end{tabular}

Participants reported some degree of cognitive load required to operate system (mean 2.19, SD 1.14) and moderate level of needs for prior learning to proper use of the program. Majority participants felt confident using program (mean 3.90, SD 0.87 ), but need for technical support was high (mean 3.90, SD 0.79). In terms of google glass, perceived physical efforts for using device was low (mean 1.96, SD 1.06) with little fatigue on head

(mean 1.55, SD 0.72) or arms (mean 1.42, SD 0.72). Participants were more critical for display caused fatigue in eyes (mean 2.19, SD 1.17).

Table 5. Fidelity, immersion and presence of smart-glass based TBS 


\begin{tabular}{|c|c|c|c|c|c|c|}
\hline Fidelity of simulation & $\begin{array}{l}\text { Mean } \pm S D \\
(1-5)\end{array}$ & $\begin{array}{l}\text { Strongly } \\
\text { Disagree } \\
\mathrm{n}(\%)\end{array}$ & $\begin{array}{l}\text { Disagree } \\
\mathrm{n}(\%)\end{array}$ & $\begin{array}{l}\text { Neither } \\
\mathrm{n}(\%)\end{array}$ & $\begin{array}{l}\text { Agree } \\
\mathrm{n}(\%)\end{array}$ & $\begin{array}{l}\text { Strongly } \\
\text { agree } \\
\mathrm{n}(\%)\end{array}$ \\
\hline $\begin{array}{l}\text { The tasks within simulation } \\
\text { were too simplified }\end{array}$ & $2.00 \pm 1.00$ & $\begin{array}{l}11 \\
(35.5)\end{array}$ & $\begin{array}{l}12 \\
(38.7)\end{array}$ & $\begin{array}{l}6 \\
(19.4)\end{array}$ & $\begin{array}{l}1 \\
(3.2)\end{array}$ & $\begin{array}{l}1 \\
(3.2)\end{array}$ \\
\hline $\begin{array}{l}\text { The simulation behaved in a } \\
\text { very unusual manner }\end{array}$ & $2.00 \pm 1.21$ & $\begin{array}{l}14 \\
(45.2)\end{array}$ & $\begin{array}{l}9 \\
(29.0)\end{array}$ & $\begin{array}{l}4 \\
(12.9)\end{array}$ & $\begin{array}{l}2 \\
(6.5)\end{array}$ & $\begin{array}{l}2 \\
(6.5)\end{array}$ \\
\hline $\begin{array}{l}\text { The contents provided via } \\
\text { smart-glass was not helpful }\end{array}$ & $2.03 \pm 0.98$ & $\begin{array}{l}11 \\
(35.5)\end{array}$ & $\begin{array}{l}11 \\
(35.5)\end{array}$ & $\begin{array}{l}6 \\
(19.4)\end{array}$ & $\begin{array}{l}3 \\
(9.7)\end{array}$ & \\
\hline $\begin{array}{l}\text { The information provided via } \\
\text { smart glass was well used } \\
\text { within the simulation }\end{array}$ & $4.13 \pm 0.67$ & & $\begin{array}{l}5 \\
(16.1)\end{array}$ & $\begin{array}{l}17 \\
(54.8)\end{array}$ & $\begin{array}{l}9 \\
(29.0)\end{array}$ & \\
\hline $\begin{array}{l}\text { The information provided via } \\
\text { smart glass was useful for } \\
\text { problem solving }\end{array}$ & $4.26 \pm 0.68$ & & $\begin{array}{l}4 \\
(12.9)\end{array}$ & $\begin{array}{l}15 \\
(48.4)\end{array}$ & $\begin{array}{l}12 \\
(38.7)\end{array}$ & \\
\hline $\begin{array}{l}\text { The quality of graphics was } \\
\text { very realistic }\end{array}$ & $3.48 \pm 0.89$ & & $\begin{array}{l}4 \\
(12.9)\end{array}$ & $\begin{array}{l}12 \\
(38.7)\end{array}$ & $\begin{array}{l}11 \\
(35.5)\end{array}$ & $\begin{array}{l}4 \\
(12.9)\end{array}$ \\
\hline $\begin{array}{l}\text { The quality of graphics } \\
\text { influenced my performance }\end{array}$ & $3.26 \pm 1.18$ & $\begin{array}{l}3 \\
(9.7)\end{array}$ & $\begin{array}{l}5 \\
(16.1)\end{array}$ & $\begin{array}{l}8 \\
(25.8)\end{array}$ & $\begin{array}{l}11 \\
(35.5)\end{array}$ & $\begin{array}{l}4 \\
(12.9)\end{array}$ \\
\hline $\begin{array}{l}\text { I thought that AR contents were } \\
\text { properly aligned with real ones }\end{array}$ & $3.74 \pm 0.77$ & & $\begin{array}{l}1 \\
(3.2)\end{array}$ & $\begin{array}{l}11 \\
(35.5)\end{array}$ & $\begin{array}{l}14 \\
(45.2)\end{array}$ & $\begin{array}{l}5 \\
(16.1)\end{array}$ \\
\hline Immersion/Presence & & & & & & \\
\hline $\begin{array}{l}\text { The information provided by the } \\
\text { smart-glass gave me the } \\
\text { impression of being somewhere } \\
\text { else }\end{array}$ & $2.19 \pm 1.01$ & $\begin{array}{l}8 \\
(25.8)\end{array}$ & $\begin{array}{l}13 \\
(41.9)\end{array}$ & $\begin{array}{l}7 \\
(22.6)\end{array}$ & $\begin{array}{l}2 \\
(6.5)\end{array}$ & $\begin{array}{l}1 \\
(3.2)\end{array}$ \\
\hline $\begin{array}{l}\text { I was really engaged to the } \\
\text { simulation }\end{array}$ & $3.77 \pm 0.84$ & $\begin{array}{l}1 \\
(3.2)\end{array}$ & & $\begin{array}{l}9 \\
(29.0)\end{array}$ & $\begin{array}{l}16 \\
(51.6)\end{array}$ & $\begin{array}{l}5 \\
(16.1)\end{array}$ \\
\hline $\begin{array}{l}\text { The quality of the graphics } \\
\text { reduced the level of immersion } \\
\text { / sense of presence }\end{array}$ & $2.16 \pm 1.10$ & $\begin{array}{l}9 \\
(29.0)\end{array}$ & $\begin{array}{l}14 \\
(45.2)\end{array}$ & $\begin{array}{l}3 \\
(9.7)\end{array}$ & $\begin{array}{l}4 \\
(12.9)\end{array}$ & $\begin{array}{l}1 \\
(3.2)\end{array}$ \\
\hline $\begin{array}{l}\text { The display field-of-view } \\
\text { reduced the level of immersion } \\
\text { / sense of presence }\end{array}$ & $2.29 \pm 1.13$ & $\begin{array}{l}8 \\
(25.8)\end{array}$ & $\begin{array}{l}12 \\
(38.7)\end{array}$ & $\begin{array}{l}7 \\
(22.6)\end{array}$ & $\begin{array}{l}2 \\
(6.5)\end{array}$ & $\begin{array}{l}2 \\
(6.5)\end{array}$ \\
\hline
\end{tabular}


The study participants reported good usability of information provided via smart-glass (mean 4.13, SD 0.67 ) and usefulness for problem solving within simulation scenarios. Four participants (13\%) reported lack of realism of the current smart-glass based TBS.

Except for one study (3.2\%), the most participants expressed that they could well engaged to the simulation.

Table 6. Likability of smart-glass based TBS program $(n=31)$

\begin{tabular}{|c|c|c|c|c|c|c|}
\hline Likability & $\begin{array}{l}\text { Mean } \pm S D \\
(1-5)\end{array}$ & $\begin{array}{l}\text { Strongly } \\
\text { Disagree } \\
n(\%)\end{array}$ & $\begin{array}{l}\text { Disagree } \\
\mathrm{n}(\%)\end{array}$ & $\begin{array}{l}\text { Neither } \\
\mathrm{n}(\%)\end{array}$ & $\begin{array}{l}\text { Agree } \\
\mathrm{n}(\%)\end{array}$ & $\begin{array}{l}\text { Strongly } \\
\text { agree } \\
\mathrm{n}(\%)\end{array}$ \\
\hline $\begin{array}{l}\text { The smart-glass based TBS is } \\
\text { pleasant }\end{array}$ & $4.65 \pm 0.55$ & & & $\begin{array}{l}1 \\
(3.2)\end{array}$ & $\begin{array}{l}9 \\
(29.0)\end{array}$ & $\begin{array}{l}21 \\
(67.7)\end{array}$ \\
\hline $\begin{array}{l}\text { I enjoyed smart-glass based } \\
\text { TBS. }\end{array}$ & $4.39 \pm 0.76$ & & $\begin{array}{l}1 \\
(3.2)\end{array}$ & $\begin{array}{l}2 \\
(6.5)\end{array}$ & $\begin{array}{l}12 \\
(38.7)\end{array}$ & $\begin{array}{l}16 \\
(51.6)\end{array}$ \\
\hline $\begin{array}{l}\text { I would participate this kind of } \\
\text { simulation again }\end{array}$ & $4.77 \pm 0.42$ & & & & $\begin{array}{l}7 \\
(22.6)\end{array}$ & $\begin{array}{l}24 \\
(77.4)\end{array}$ \\
\hline $\begin{array}{l}\text { I could see practical benefits of } \\
\text { this program in health care } \\
\text { settings }\end{array}$ & $4.52 \pm 0.62$ & & & $\begin{array}{l}2 \\
(6.5)\end{array}$ & $\begin{array}{l}11 \\
(35.5)\end{array}$ & $\begin{array}{l}18 \\
(58.1)\end{array}$ \\
\hline $\begin{array}{l}\text { Smart-glass based team } \\
\text { simulation is an effective } \\
\text { educational strategy }\end{array}$ & $4.52 \pm 0.76$ & & $\begin{array}{l}1 \\
(3.2)\end{array}$ & $\begin{array}{l}2 \\
(6.5)\end{array}$ & $\begin{array}{l}8 \\
(25.8)\end{array}$ & $\begin{array}{l}20 \\
(64.5)\end{array}$ \\
\hline $\begin{array}{l}\text { Smart-glass based team } \\
\text { simulation is helpful for my } \\
\text { future clinical practice }\end{array}$ & $4.58 \pm 0.67$ & & & $\begin{array}{l}3 \\
(9.7)\end{array}$ & $\begin{array}{l}7 \\
(22.6)\end{array}$ & $\begin{array}{l}21 \\
(67.7)\end{array}$ \\
\hline $\begin{array}{l}\text { Smart-glass based team } \\
\text { simulation meets my } \\
\text { educational needs }\end{array}$ & $4.13 \pm 0.99$ & & $\begin{array}{l}2 \\
(6.5)\end{array}$ & $\begin{array}{l}7 \\
(22.6)\end{array}$ & $\begin{array}{l}7 \\
(22.6)\end{array}$ & $\begin{array}{l}15 \\
(48.4)\end{array}$ \\
\hline
\end{tabular}

Majority participants reported that they had pleasant experience (mean 4.65, SD 0.55) and have intention to use this kind of simulation again (mean 4.77, SD 0.42). Most participants agreed that the smart glass had high potential to produce practical benefits (mean 4.52, SD 0.62) and also effective in simulation education (mean 4.52, SD 0.76).

Table 7. User satisfaction of smart glass based TBS program $(n=31)$ 


\begin{tabular}{|llllll|}
\hline & $\begin{array}{l}\text { Mean } \pm \text { SD } \\
(1-4)\end{array}$ & $\begin{array}{l}\text { Very } \\
\text { unsatisfied } \\
\mathrm{n}(\%)\end{array}$ & $\begin{array}{l}\text { Unsatisfied } \\
(\%)\end{array}$ & $\begin{array}{l}\text { Satisfied } \\
\mathrm{n}(\%)\end{array}$ & $\begin{array}{l}\text { Very } \\
\text { satisfied } \\
\mathrm{n}(\%)\end{array}$ \\
\hline Smart-glass device & $2.81 \pm 0.83$ & $2(6.5)$ & $8(25.8)$ & $\begin{array}{l}15 \\
(48.4)\end{array}$ & $6(19.4)$ \\
\hline $\begin{array}{l}\text { Easy of use of smart- } \\
\text { glass }\end{array}$ & $2.94 \pm 0.77$ & $1(3.2)$ & $7(22.6)$ & $\begin{array}{l}16 \\
(51.6)\end{array}$ & $7(22.6)$ \\
\hline $\begin{array}{l}\text { System output } \\
\text { Smart-glass based TBS }\end{array}$ & $2.68 \pm 0.94$ & $4(12.9)$ & $8(25.8)$ & $\begin{array}{l}13 \\
(41.9)\end{array}$ & $6(19.4)$ \\
\hline
\end{tabular}

Participants' level of satisfaction that highest scores were obtained on smart-glass based TBS (mean 3.26, SD 0.68 ) and system output were reported as lowest satisfaction (mean 2.68, SD 0.94).

Table 8. Attitude toward interprofessional health care team $(n=31)$

\begin{tabular}{|llll|}
\hline & Category & Mean & SD \\
\hline $\begin{array}{l}\text { Attitudes towards Interprofessional Health Care Team } \\
\text { (ATIHCT) }\end{array}$ & Quality of care & 4.34 & 0.61 \\
\cline { 2 - 3 } & $\begin{array}{l}\text { Person centered } \\
\text { care }\end{array}$ & 4.26 & 0.64 \\
\cline { 2 - 3 } & Time constraints & 3.91 & 0.77 \\
\cline { 2 - 3 } & Total score & 4.23 & 0.59 \\
\hline
\end{tabular}

Table 9. Learning satisfaction of TBS $(n=31)$ 


\begin{tabular}{|lll|}
\hline Item & Mean & SD \\
\hline It was interesting & 8.71 & 1.40 \\
\hline It is effective way to achieve learning goal & 8.58 & 1.46 \\
\hline It provided meaningful learning experience & 9.03 & 1.22 \\
\hline It is more effective than theory-based lecture & 8.81 & 1.20 \\
\hline I actively engaged in learning activities & 9.19 & 1.08 \\
\hline I am satisfied with the class overall & 9.10 & 1.38 \\
\hline I would like to participate other smart-glass based TBS & 9.06 & 1.29 \\
\hline Learning satisfaction total & 62.48 & 7.30 \\
\hline
\end{tabular}

Overall, the attitude towards interprofessional health care team was scored 4.23 (SD 0.59) and participants scored 'quality of care 'subscale relatively higher than other subscales (Table 8). The table 9 showed the learning satisfaction of current smart-glass based TBS. In terms of learning satisfaction, the item "I actively engaged in learning activities" scored the highest (mean 9.19, SD 1.08) and the item "It is effective way to achieve learning goal" scored the lowest (mean 8.58, SD 1.46).

\section{Qualitative response}

\section{Overall experience}

About eight out of ten (84\%) participants responded positively. Users reported that smart glasses-based team simulation was new, exciting, and interesting (40\%) and experienced practical assistance while using the smart glasses during the simulation (44\%). Some participants (16\%) felt frustrated, reporting that the system needed further improvement.

\section{Perceived benefits}

Thirty participants stated that they expected that smart glasses would improve the accuracy of nursing skill performance using image and voice guidance (96.8\%). Seventeen users (54.8\%) reported that fast and accurate information exchange using smart glasses could assist interprofessional teamwork.

Twenty-six participants (83.9\%) reported the system could prevent the occurrence of errors saying, "The remote supporter found me using the wrong dose of medication." Six participants (19.4\%) reported they felt confident using medical devices saying, "Although I used EKG device for the first time, image guidance and voice assistance from the remote supporter was really helpful."

Recommendation 
The study participants' recommendations for further improvements were largely about the narrow field of view provided for remote supporters (46\%), lack of smoothness in communication between remote supporters and bedside trainees (45\%), and discomfort of the smart glasses, especially for those who wear glasses (13\%). When taking the role of remote supporter, participants complained that "It was sometimes hard to figure out the patient's condition. I guess it might be easier with real patients, but it was really difficult when a mannequin was the patient." In terms of communication, most problems were caused by incomplete construction of the network and Bluetooth earphones that participants reported as "Wearing glass and earphones at the same time seems too much. It is needed to have something that can replace earphones." In addition, users recommended adding a visual status indicator to confirm whether the right information was sent $(n=2,6.5 \%)$, suggesting "It would be nicer to confirm whether the image and text that I clicked and typed were sent. I had to keep asking what bedside trainees were seeing. $"$

The present study evaluated the usability and feasibility of adopting smart glasses for training of nursing skills and interprofessional interactions in emergency arrhythmia situations. The findings of this study indicate that participants attempted to use various features of the smart glasses to be actively engaged in team working and clinical decision making to provide the best nursing care. The results of the current study provide evidence of the great potential for the use of smart glasses in clinical settings for interprofessional interactions.

The majority of students perceived TBS using smart glasses as a new, exciting, and interesting method for learning. This is in line with previous studies that the incorporation of high-tech devices into education could promote great interest among this digitally native generation [24]. Given the clear roles within the simulation (either bedside trainees or remote supporters), the chances were greater for participants to take part in team communication, with greater responsibility on the remote supporters.

Although the scores were higher in the smart glasses-based TBS group, the results of this study showed no statistically significant group differences in attitude towards interprofessional healthcare teams. Among the subscales, the scores for time constraints and person-centered care showed relatively greater differences. By being constantly connected to the remote supporters, bedside trainees felt more comfortable seeking external help which users found to be a way to address issues of time constraints within team work. In fact, most features of the smart glasses allow for the seamless flow of teamwork [25], which could be a great advantage in emergency care settings where efficient and accurate interprofessional interactions are key to better patient outcomes.

Generally, the users responded positively regarding the GG itself, expressing good feasibility for long-term use with a low level of physical discomfort experienced. One explanation may be that the weight of the GG EE2 is $46 \mathrm{~g}$, which is relatively lighter than other smart glasses (65-350 g) [26]. However, no previous studies have clearly reported wearability and comfortability of the GG; thus, further comparison studies could contribute to the physical design of wearable devices. 
Interestingly, users who experienced discomfort were mostly related to the accessories, and only a few participants from previous studies complained regarding the physical aspects of the GG (short battery life, heavy weight, etc.) [27-28]. We used Bluetooth earphones and mirrors to compensate for the defects of the smart glasses, which users found to be superfluous. There was consistent frustration expressed by individuals wearing glasses, reporting discomfort of the double layering of glasses. Although one of the reasons why the GG was chosen was the lens-free design, it would be better to seek other solutions.

The findings of this study revealed that the participants were least satisfied with the system output asking for better resolution and bigger screens. Indeed, wearable assistive technologies have different intended use, environment, and trade-offs [29-30]. In clinical settings, assistive devices should enable the wearer to seamlessly follow clinical workflows of the main task, benefit from hands-free device potential, and maintain sterile conditions [31]. The GG's small display limits the wearer's peripheral vision and multitasking capabilities [32] to keep them more focused and attentive to clinical tasks and workflows. In this study, the use of inexperienced devices within a tense situation could aggravate the difficulties related to the display; thus, it is suggested that sufficient time is provided for users to become accustomed to new devices.

Perceived usefulness of smart glass based TBS for future clinical practice was great among study participants. The current system was designed to focus on the exchange of information between remote supporters and bedside trainees and knowing the high risks of medical errors from unskilled care providers [33], the participants of this study found the smart glasses to be of great use for interprofessional teamwork. Previous studies also showed that the risk signs of patients were better detected when sharing patient monitoring via smart glasses [28].

The participants reported low complexity of the simulation program, but some users complained of low compliance. Delayed audio was reported as an important concern that should be resolved. A previous study identified the requirements of low-latency applications for virtual reality (latency $=1 \mathrm{~ms}$ ) and telesurgery (1-10 ms) [34]. Although there were concerns over network issue, this is an area where technology is growing rapidly. Therefore, it is worthwhile for the development additional smart glassesbased interventions for education and clinical use.

Further consideration of user-centered interfaces may be the key to success in future system development. The principles of user-friendly interface design include understanding the intended users and their needs [35]. Users recommended the additional incorporation of visual status indicators due to the degree of uncertainty related to communication that was experienced within the current system. As this system was designed for use in emergency care settings, it is necessary to minimize the cognitive effort of users to verify their actions.

\section{Limitation}

This study has some limitations. It was difficult to draw clear conclusions by relying on self-reported questionnaires. In addition, the quasi-experimental design for the two-outcome measures did not 
sufficiently prove the effectiveness of the smart glasses-based TBS in nursing education.

\section{Discussion}

The present study evaluated the usability and feasibility of adopting smart glasses for training of nursing skills and interprofessional interactions in emergency arrhythmia situations. The findings of this study indicate that participants attempted to use various features of the smart glasses to be actively engaged in team working and clinical decision making to provide the best nursing care. The results of the current study provide evidence of the great potential for the use of smart glasses in clinical settings for interprofessional interactions.

The majority of students perceived TBS using smart glasses as a new, exciting, and interesting method for learning. This is in line with previous studies that the incorporation of high-tech devices into education could promote great interest among this digitally native generation [24]. Given the clear roles within the simulation (either bedside trainees or remote supporters), the chances were greater for participants to take part in team communication, with greater responsibility on the remote supporters.

Although the scores were higher in the smart glasses-based TBS group, the results of this study showed no statistically significant group differences in attitude towards interprofessional healthcare teams. Among the subscales, the scores for time constraints and person-centered care showed relatively greater differences. By being constantly connected to the remote supporters, bedside trainees felt more comfortable seeking external help which users found to be a way to address issues of time constraints within team work. In fact, most features of the smart glasses allow for the seamless flow of teamwork [25], which could be a great advantage in emergency care settings where efficient and accurate interprofessional interactions are key to better patient outcomes.

Generally, the users responded positively regarding the GG itself, expressing good feasibility for long-term use with a low level of physical discomfort experienced. One explanation may be that the weight of the GG EE2 is $46 \mathrm{~g}$, which is relatively lighter than other smart glasses (65-350 g) [26]. However, no previous studies have clearly reported wearability and comfortability of the GG; thus, further comparison studies could contribute to the physical design of wearable devices.

Interestingly, users who experienced discomfort were mostly related to the accessories, and only a few participants from previous studies complained regarding the physical aspects of the GG (short battery life, heavy weight, etc.) [27-28]. We used Bluetooth earphones and mirrors to compensate for the defects of the smart glasses, which users found to be superfluous. There was consistent frustration expressed by individuals wearing glasses, reporting discomfort of the double layering of glasses. Although one of the reasons why the GG was chosen was the lens-free design, it would be better to seek other solutions.

The findings of this study revealed that the participants were least satisfied with the system output asking for better resolution and bigger screens. Indeed, wearable assistive technologies have different intended use, environment, and trade-offs [29-30]. In clinical settings, assistive devices should enable the 
wearer to seamlessly follow clinical workflows of the main task, benefit from hands-free device potential, and maintain sterile conditions [31]. The GG's small display limits the wearer's peripheral vision and multitasking capabilities [32] to keep them more focused and attentive to clinical tasks and workflows. In this study, the use of inexperienced devices within a tense situation could aggravate the difficulties related to the display; thus, it is suggested that sufficient time is provided for users to become accustomed to new devices.

Perceived usefulness of smart glass based TBS for future clinical practice was great among study participants. The current system was designed to focus on the exchange of information between remote supporters and bedside trainees and knowing the high risks of medical errors from unskilled care providers [33], the participants of this study found the smart glasses to be of great use for interprofessional teamwork. Previous studies also showed that the risk signs of patients were better detected when sharing patient monitoring via smart glasses [28].

The participants reported low complexity of the simulation program, but some users complained of low compliance. Delayed audio was reported as an important concern that should be resolved. A previous study identified the requirements of low-latency applications for virtual reality (latency $=1 \mathrm{~ms}$ ) and telesurgery $(1-10 \mathrm{~ms})$ [34]. Although there were concerns over network issue, this is an area where technology is growing rapidly. Therefore, it is worthwhile for the development additional smart glasses-based interventions for education and clinical use.

Further consideration of user-centered interfaces may be the key to success in future system development. The principles of user-friendly interface design include understanding the intended users and their needs [35]. Users recommended the additional incorporation of visual status indicators due to the degree of uncertainty related to communication that was experienced within the current system. As this system was designed for use in emergency care settings, it is necessary to minimize the cognitive effort of users to verify their actions.

Limitation

This study has some limitations. It was difficult to draw clear conclusions by relying on self-reported questionnaires. In addition, the quasi-experimental design for the two-outcome measures did not sufficiently prove the effectiveness of the smart glasses-based TBS in nursing education.

\section{Conclusion}

Smart glasses were introduced as an effective means of communication in the industry. In this study, we presented a TBS using GG to investigate the usability and feasibility of smart glasses for interprofessional interaction. Using the scenario of patients with arrhythmia, students took the role of bedside trainees and remote supporters and used the GG for knowledge sharing and communication. The findings of this study indicate that the current program has great potential for simulation environments 
as well for clinical settings. Some drawbacks were identified, which require further consideration and improvement in future testing.

\section{Declarations}

\section{Ethics approval and consent to participate}

The procedures of this study were approved by the review board of M University in Korea (IRB No. MNUIRB-201006-SB-011-02). Written informed consent was obtained from study participants before the commencement of study.

\section{Competing interests}

The authors declare that this work has no competing interest.

\section{Funding}

This research was supported by a grant (20012234) of Regional Customized Disaster-Safety R\&D Program funded by the Ministry of Interior and Safety (MOIS, Korea). This work was supported by a National Research Foundation of Korea (NRF) grant funded by the Korea government (MSIT) (No. NRF2018R1D1A1B07048247, No. NRF-2018R1D1A1B07043983).

\section{References}

[1] W. J. McKenna, B. J. Maron, C. Thiene. Classification, epidemiology, and global burden of cardiomyopathies, Circ. Res., 121 (7) (2017), pp. 722-730, https://doi.org/10.1161/CIRCRESAHA.117.309711

[2] M. O. Bekkink, S. E. Farrell, J. K. Takayesu. Interprofessional communication in the emergency department: residents' perceptions and implications for medical education. Int. J. Med. Educ., 9 (2018), pp. 262-270, https://doi.org/10.5116/ijme.5bb5.c111

[3] L. Bazrafkan, M. Hemmati. The effect of Cardiac Arrhythmias Simulation Software on the nurses' learning and professional development. J. Adv. Med. Educ. Prof., 6 (2) (2018), pp. 86-91.

[4] J. G. Andrade, L. Macle, S. Nattel, A. Verma, J. Cairns. Contemporary atrial fibrillation management: A comparison of the current AHA/ACC/HRS, CCS, and ESC guidelines. Can. J. Cardiol., 33 (8) (2017), pp. 965-976, https://doi.org/10.1016/j.cjca.2017.06.002

[5] J. De Bie, C. Martignani, G. Massaro, I. Diemberger. Performance of seven ECG interpretation programs in identifying arrhythmia and acute cardiovascular syndrome. J. Electrocardiol., 58 (2020), pp. 143-149, https://doi.org/10.1016/j.jelectrocard.2019.11.043 
[6] C. La Cerra, A. Dante, V. Caponnetto, I. Franconi, E. Gaxhja, C. Petrucci, L. Lancia. Effects of high-fidelity simulation based on life-threatening clinical condition scenarios on learning outcomes of undergraduate and postgraduate nursing students: A systematic review and meta-analysis. BMJ open, 9 (2) (2019), e025306, https://doi.org/10.1136/bmjopen-2018-025306

[7] S. Armenia, L. Thangamathesvaran, A. D. Caine, N. King, A. Kunac, A. M. Merchant. The role of highfidelity team-based simulation in acute care settings: A systematic review. Surg. J. (N Y), 4 (3) (2018), pp. e136-e151, https://doi.org/10.1055/s-0038-1667315

[8] J. H. Yoon, E. J. Lee. The effect of team based simulation learning using SBAR on critical thinking and communication clarity of nursing students. J. Korea Acad.-Ind. Coop. Soc., 19 (9) (2018), pp. 42-49, https://doi.org/10.5762/KAIS.2018.19.9.42

[9] D. J. W. Reed, R. L. Hermelin, C. S. Kennedy, J. Sharma. Interdisciplinary onsite team-based simulation training in the neonatal intensive care unit: a pilot report. J. Perinatol., 37 (4) (2017), pp. 461-464, https://doi.org/10.1038/jp.2016.238

[10] H. Y. Kang, E. Y. Choi, H. R. Kim. Nursing student's experiences in team based simulation learning. J. Korean Acad. Soc. Nurs. Educ., 19 (1) (2013), pp. 5-15, https://doi.org/10.5977/jkasne.2013.19.1.5

[11] M. Nasajpour, S. Pouriyeh, R. M. Parizi, M. Dorodchi, M. Valero, H. R. Arabnia. Internet of things for current COVID-19 and future pandemics: An exploratory study. J. Healthc. Inform. Res., 4 (2020), pp. 325364, https://doi.org/10.1007/s41666-020-00080-6

[12] S. Carreiro, P. R. Chai, J. Carey, B. Chapman, E. W. Boyer. Integrating personalized technology in toxicology: Sensors, smart glass, and social media applications in toxicology research. J. Med. Toxicol., 13 (2) (2017), pp. 166-172, https://doi.org/10.1007/s13181-017-0611-y

[13] V. Ponnusamy, A. Coumaran, A. S. Shunmugam, K. Rajaram, S. Senthilvelavan. Smart glass: Realtime leaf disease detection using YOLO transfer learning. 2020 International Conference on Communication and Signal Processing (ICCSP), Chennai, India, (2020), pp. 1150-1154, https://doi.org/10.1109/ICCSP48568.2020.9182146

[14] F. Demir, S. Ahmad, P. Calyam, D. Jiang, R. Huang, I. Jahnke. A next-generation augmented reality platform for Mass Casualty Incidents (MCl). J. Usability Stud., 12 (4) (2017), pp. 193-214.

[15] H. Wüller, J. Behrens, M. Garthaus, S. Marquard, H. Remmers. A scoping review of augmented reality in nursing. BMC Nurs., 18 (2019), 19, https://doi.org/10.1186/s12912-019-0342-2

[16] I. Radu. Augmented reality in education: a meta-review and cross-media analysis. Pers. Ubiquitous Comput., 18 (6) (2014), pp. 1533-1543, https://doi.org/10.1007/s00779-013-0747-y

[17] B. M. Garrett, C. Jackson, B. Wilson. Augmented reality m-learning to enhance nursing skills acquisition in the clinical skills laboratory. Interact. Technol. Smart Educ., 12 (4) (2015), pp. 298-314, 
[18] J. Vaughn, M. Lister, R. J. Shaw. Piloting augmented reality technology to enhance realism in clinical simulation. Comput. Inform. Nurs., 34 (9) (2016), pp. 402-405, https://doi.org/10.1097/CIN.0000000000000251

[19] E. S. Binstadt, R. M. Walls, B. A. White, E. S. Nadel, J. K. Takayesu, T. D. Barker, C. N. Pozner. A comprehensive medical simulation education curriculum for emergency medicine residents. Ann. Emerg. Med., 49 (4) (2007), pp. 495-504, https://doi.org/10.1016/j.annemergmed.2006.08.023

[20] P. L. Ingrassia, G. Mormando, E. Giudici, F. Strada, F. Carfagna, F. Lamberti, A. Bottino. Augmented reality learning environment for basic life support and defibrillation training: usability study. J. Med. Internet Res., 22 (5) (2020), e14910, https://doi.org/10.2196/14910.

[21] G. D. Heinemann, M. H. Schmitt, M. P. Farrell, S. A. Brallier. Development of an attitudes toward health care teams scale. Eval. Health Prof., 22 (1) (1999), pp. 123-142,

https://doi.org/10.1177/01632789922034202

[22] V. R. Curran, D. Sharpe, J. Forristall, K. Flynn. Attitudes of health sciences students towards interprofessional teamwork and education. Learn. Health Soc. Care, 7 (3) (2008), pp. 146-156, https://doi.org/10.1111/j.1473-6861.2008.00184.x

[23] Y. R. Ji, H. M. Chung. Effects of case-based learning on task achievement and learning satisfaction in the university class. J. Learner-Cent. Curriculum Instr., 14 (9) (2014), pp. 243-265.

[24] C. Moro, Z. Štromberga, A. Raikos, A. Stirling. The effectiveness of virtual and augmented reality in health sciences and medical anatomy. Anat. Sci. Educ., 10 (6) (2017), pp. 549-559, https://doi.org/10.1002/ase.1696

[25] E. Chew, P. L. Lee, W. H. Ho. Enhancing seamless nurses-physician communication after-hours with Google Glass. 2018 International Conference on Smart Computing and Electronic Enterprise (ICSCEE), Shah Alam, (2018), pp. 1-6, https://doi.org/10.1109/ICSCEE.2018.8538415

[26] A. Syberfeldt, O. Danielsson, P. Gustavsson. Augmented reality smart glasses in the smart factory: Product evaluation guidelines and review of available products. IEEE Access, 5 (2017), pp. 9118-9130, https://doi.org/10.1109/ACCESS.2017.2703952

[27] B. Chaballout, M. Molloy, J. Vaughn, R. Brisson lii, R. Shaw. Feasibility of augmented reality in clinical simulations: using Google glass with manikins. JMIR Med. Educ., 2 (1) (2016), e2, https://doi.org/10.2196/mededu.5159

[28] M. H. Iqbal, A. Aydin, A. Lowdon, H. I. Ahmed, G. H. Muir, M. S. Khan, K. Ahmed. The effectiveness of Google GLASS as a vital signs monitor in surgery: A simulation study. Int. J. Surg., 36 (Pt A) (2016), pp. 293-297, https://doi.org/10.1016/j.ijsu.2016.11.01 
[29] E. M. Benssassi, J. C. Gomez, L .E. Boyd, G. R. Hayes, J. Ye. Wearable assistive technologies for autism: Opportunities and challenges. IEEE Pervasive Comput., 17 (2) (2018), pp. 11-21, https://doi.org/10.1109/mprv.2018.022511239

[30] M. Zheng, P. X. Liu, R. Gravina, G. Fortino. An emerging wearable world: New gadgetry produces a rising tide of changes and challenges. IEEE Syst. Man Cybern. Mag., 4 (4) (2018), pp. 6-14, https://doi.org/10.1109/MSMC.2018.2806565

[31] N. J. Wei, B. Dougherty, A. Myers, S. M. Badawy. Using Google Glass in surgical settings: systematic review. JMIR MHealth UHealth, 6 (3) (2018), e54, https://doi.org/10.2196/mhealth.9409

[32] I. Chaturvedi, F. H. Bijarbooneh, T. Braud, P. Hui. Peripheral vision: a new killer app for smart glasses. Proceedings of the 24th International Conference on Intelligent User Interfaces (IUI '19), New York, USA, (2019), pp. 625-636, https://doi.org/10.1145/3301275.3302263

[33] A. Avillion. Medical errors: A critical practice issue. CE for lowa Nursing Professionals, (2017), pp. 130-141.

[34] X. Jiang, H. Shokri-Ghadikolaei, G. Fodor, E. Modiano, Z. Pang, M. Zorzi, C. Fischione. Low-latency networking: Where latency lurks and how to tame it. Proc. IEEE, 107 (2) (2019), pp. 280-306, https://doi.org/10.1109/JPROC.2018.2863960

[35] B. Sedlmayr, J. Schöffler, H. U. Prokosch, M. Sedlmayr. User-centered design of a mobile medication management. Inform. Health Soc. Care, 44 (2) (2019), pp. 152-163, https://doi.org/10.1080/17538157.2018.1437042

\section{Figures}




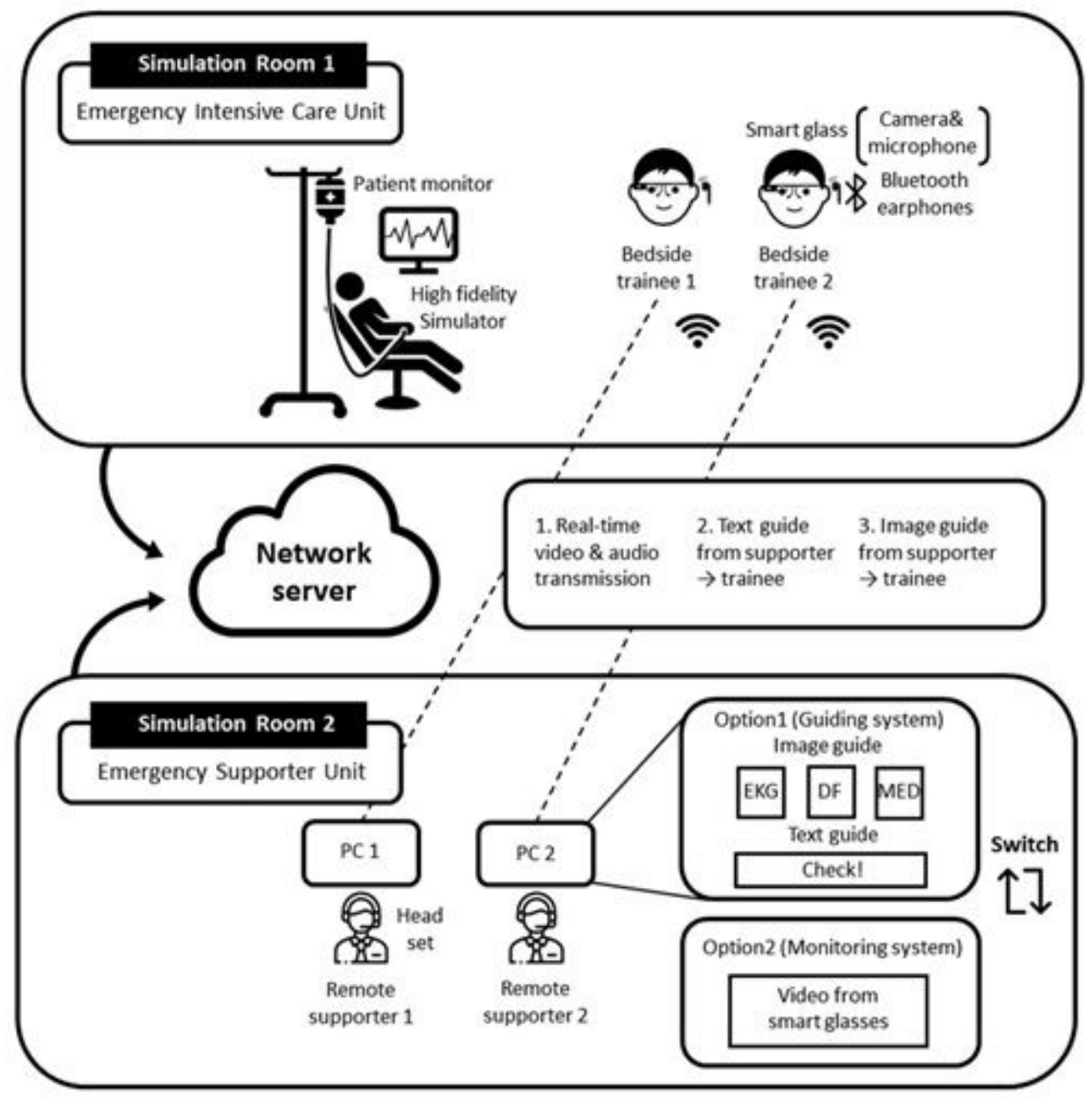

Figure 1

The flow of the team-based simulation using smart glasses and a high-fidelity simulator
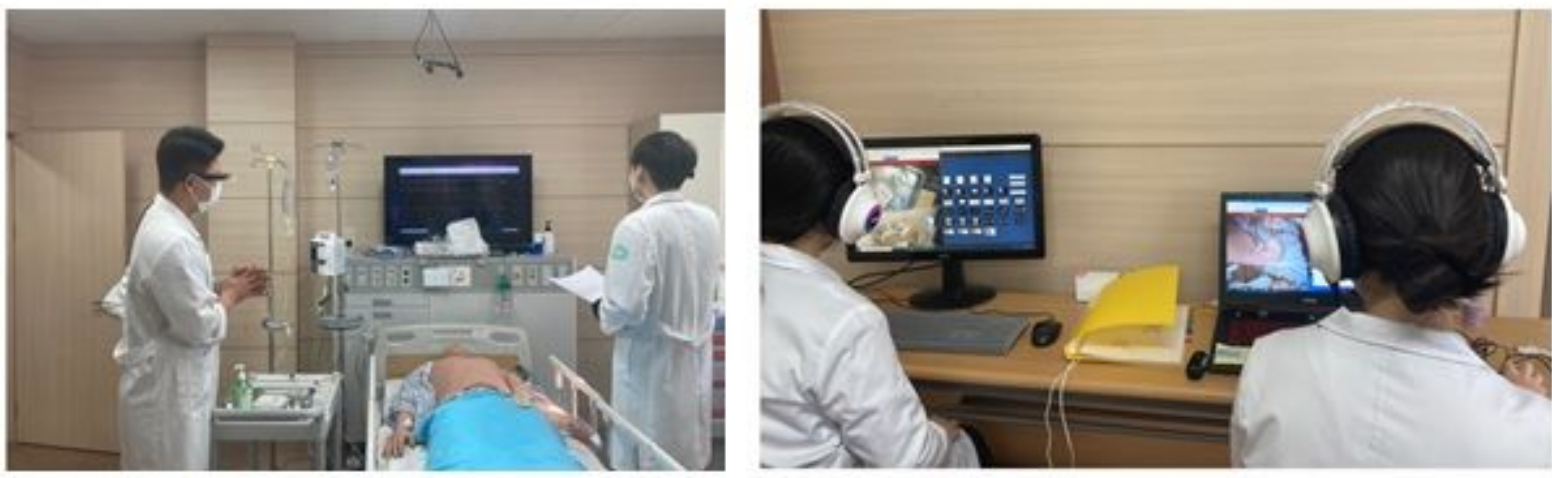

Figure 2

Room setup for emergency unit simulation training

\section{Supplementary Files}


This is a list of supplementary files associated with this preprint. Click to download.

- Appendix14.docx 